TITLE:

\title{
Long-term variation of Ampère force by geomagnetic Sq currents and thermospheric pressure difference
}

\author{
$\operatorname{AUTHOR}(\mathrm{S}):$ \\ Takeda, Masahiko
}

\section{CITATION:}

Takeda, Masahiko. Long-term variation of Ampère force by geomagnetic Sq currents and thermospheric pressure difference. Journal of Geophysical Research: Space Physics 2016, 121(11): 11407-11412

\section{ISSUE DATE:}

2016-11

URL:

http://hdl.handle.net/2433/217896

\section{RIGHT:}

Accepted for publication in 'Journal of Geophysical Research: Space Physics'. Copyright 2016 American Geophysical Union. Further reproduction or electronic distribution is not permitted.; The full-text file will be made open to the public on 16 June 2017 in accordance with publisher's 'Terms and Conditions for Self-Archiving'. 


\section{Journal of Geophysical Research: Space Physics}

\section{RESEARCH ARTICLE}

10.1002/2016JA022845

Key Points:

- Long-term variation of a neutral pressure difference can be estimated from the geomagnetic field only

- Correlation of Ampère force with solar sunspot number version 2 is slightly

higher than that with version 1

- Correlation between Ampère force and solar sunspot number is generally better than total $\mathrm{Sq}$ currents

\section{Correspondence to:}

M. Takeda,

takeda@kugi.kyoto-u.ac.jp

\section{Citation:}

Takeda, M. (2016), Long-term variation of Ampère force by geomagnetic $S q$ currents and thermospheric pressure difference, J. Geophys. Res. Space Physics, 121, 11,407-11,412, doi:10.1002/ 2016JA022845.

Received 21 APR 2016 Accepted 23 OCT 2016 Accepted article online 27 OCT 2016 Published online 12 NOV 2016

\section{Long-term variation of Ampère force by geomagnetic $S q$ currents and thermospheric pressure difference}

\author{
Masahiko Takeda ${ }^{1}$ \\ 'Data Analysis Center for Geomagnetism and Space Magnetism, Faculty of Science, Kyoto University, Kyoto, Japan
}

\begin{abstract}
The long-term variation of the Ampère force exerted by geomagnetic Sq currents was studied in relation to the thermospheric pressure. First, the long-term trend of the pressure difference was estimated using a linear regression coefficient based on a high correlation between the Ampère force and the pressure difference in recent years. The pressure difference may be inferred from solar parameters such as solar sunspot number; inspection of the difference from Sq has a merit that it gives the "response" of the thermosphere in contrast with the "input" parameters of the solar activity index. Next, the effect of the version up of sunspot number was examined. The correlation of the Ampère force with sunspot number updated in 2015 (version 2) is slightly higher than that with old sunspot number (version 1), which suggests that the later version is an improved index for the solar activity and its effect on the state of the thermosphere. Last, the correlation between total Sq currents and sunspot number was found to be less than that between the Ampère force and sunspot number, although the difference is small and could not be statistically significant. This suggests that the geomagnetic main field affects the relation between the Ampère force and the pressure difference.
\end{abstract}

\section{Introduction}

Solar quiet daily geomagnetic field variation $(S q)$ is primarily caused by the ionospheric dynamo currents that are first driven by the dynamo electric field generated by the interaction of the neutral wind with the geomagnetic main field. Fukushima [1979] showed that the Sq current system is driven by neutral winds blowing in the opposite direction to the pressure gradient. Such a wind system was previously obtained from the Sq current system by Matsushita [1969] and was shown as a simulation result for thermospheric dynamics [e.g., Miyoshi et al., 2012]. The generation of this current system requires that the neutral pressure gradient force is balanced by the Ampère force (AF) exerted by the $S q$ currents, and therefore, the neutral pressure difference (PD) between its maximum and minimum can be estimated using AF. In fact, Takeda [2015] showed that the annual averages of AF and PD are highly correlated and almost equal at the observatories around the $S q$ current vortex center. If this balance is always maintained, AF can be used as an indicator of PD, and therefore, $S q$ field variations are useful in studying the long-term variation of the thermosphere.

The long-term variation of the $S q$ field has been studied for a long time. It has been widely accepted that the amplitude of the $\mathrm{Sq}$ field is strongly controlled by solar activity [e.g., Chapman and Bartels, 1940]. This solar activity dependence is caused not by the neutral wind velocity but by the ionospheric conductivity variation, which increases ionospheric currents in a high solar activity period [Takeda, 2013]. Yamazaki and Kosch [2014] showed that both $S q$ and lunar variation $(L)$ have the same dependence on solar activity, which suggests that both are strongly controlled by the ionospheric conductivity. On the other hand, Celik [2014] studied the solar and lunar dependencies on solar sunspot number (SSN) using a very large data set and found that the dependence of $S q$, lunar variation, and $E$ region conductivity with solar cycle differ significantly from each other. This difference problem is at least partly due to the estimation of solar activity, and thus, it is a problem how solar activity can be estimated.

In addition to the solar activity dependence, many researchers examined the relations between long-term variations in the $\mathrm{Sq}$ field and several parameters such as the geomagnetic main field, geomagnetic activity, and neutral wind. For example, Glassmeier et al. [2004] examined the effects of long-term geomagnetic variation on the magnetospheric and ionospheric physics and emphasized the importance of the geomagnetic main field in studying the physics. Clilverd et al. [2005] reported that the long trend of the Sq field can be 


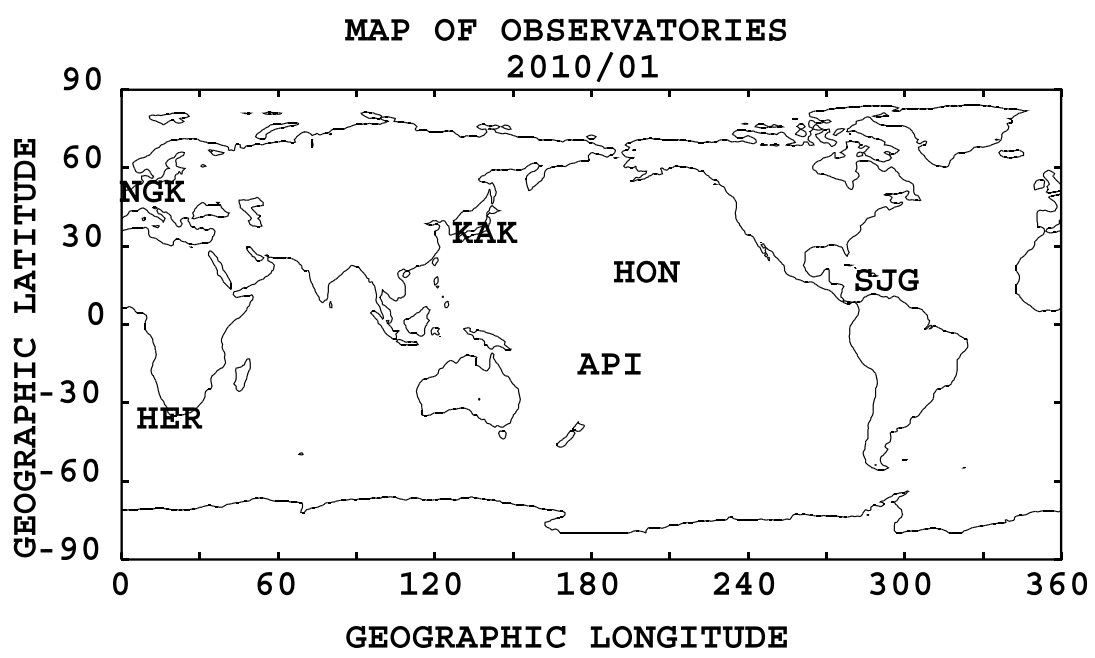

Figure 1. Geomagnetic observatories used in the present study.

partly due to solar activity. Shinbori et al. [2014] examined the residual of the Sq field removing the effects of solar activity, and the residuals showed negative values over a wide region, and an anticorrelation with geomagnetic main field intensity for about $71 \%$ of the geomagnetic stations. This anticorrelation was predicted by Takeda [1996], as the result of the enhancement of the conductivity by the reduction of the field intensity.

In most of these previous studies, the long-term trends of the thermospheric wind velocity have been examined using the $S q$ field. However, the estimation of wind velocity from the $S q$ field requires information on electric conductivity, the calculation of which needs ionospheric and thermospheric models. Furthermore, the conductivity is influenced by parameters in the thermosphere such as the density, composition, and temperature, all of which are closely related to the wind.

In the present analysis solar sunspot number (SSN) is mainly adopted as the "input" index to the Earth because it is widely use and available for a long time and compared with $S q$ field. On the other hand, the long-term variation of AF is initially estimated, and the linear regression coefficient of PD obtained from a neutral model is calculated. Next, PD is estimated from AF for the period when model PD data are not available using the obtained coefficients. Last, PD is estimated from SSN in the same method and compared with the estimation from AF. In fact, sunspot number was updated from version 1 (SSNV1) to version 2 (SSNV2) in 2015, and effect of this version up is also examined, although the most prominent change is the elimination of the 0.6 factor, but there are some modifications.

\section{Method of Analysis}

The method to determine AF is the same as that used in Takeda [2015]. That is, total meridional Sq current (TJ) is first estimated from geomagnetic hourly values of the $Y$ component between 06:00 and 18:00 LT by summing up the difference ( $Y$ (hour)) from the night value, which is the average of 21:00-02:00 LT on the day. Then, AF is estimated from the product of TJ and geomagnetic $Z$ component $\left(B_{z}\right)$, which was calculated at the $120 \mathrm{~km}$ altitude above the observatory by using International Geomagnetic Reference Field version 12 model. PD is estimated as the difference between the maximum and minimum height-integrated neutral pressures above the observatory. The height distribution of the neutral atmospheric pressure at each hour on each day was calculated using the NRLMSISE-00 atmosphere model, and the pressure was integrated from 120 to $400 \mathrm{~km}$ in altitude.

Geomagnetic observatories selected for this analysis were Hermanus (HER), San Juan (SJG), Honolulu (HON), Kakioka (KAK), Niemegk (NGK), and Apia (API), because their long-term data are relatively continuous and of good quality. The locations of these observatories are shown in Figure 1. Days when Kp index did not exceed 

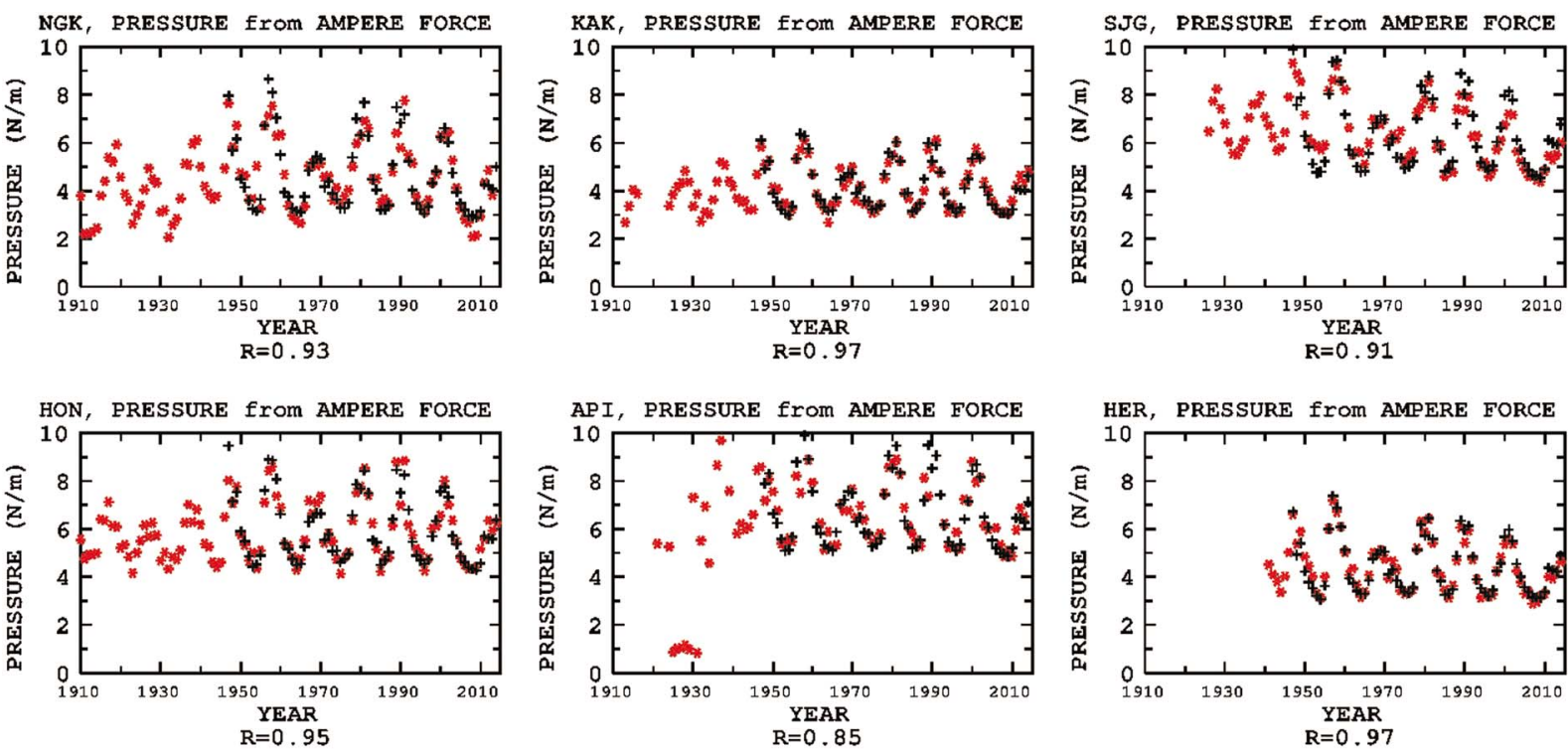

Figure 2. Model values of PD (black) and PD values (red) accessed from AF using linear regression obtained from the $1947-2010$ data. $R$ indicates the correlation coefficient.

$3+$ were used in the present analysis to avoid the geomagnetic disturbance field. Since $K p$ index is not available before 1932, the condition that aa index did not exceed 40 was adopted instead.

$\mathrm{AF}$ and PD on all quiet days during the equinox (March, April, September, and October) were averaged for each year and used in the subsequent analysis. This data selection was chosen because the effects of the interhemispheric field-aligned currents generated by the unbalance between both hemispheres are minimal at this time. To examine PD predicted using $A F$, linear regressions were applied to PD and AF for the period from 1947 to 2010 when NRLMSISE-00 atmosphere model data are available. Then PD was predicted before 1947 by using the obtained regression coefficients from AF.

Next, PD was also predicted from solar sunspot number (SSN) using the same method for comparison. SSN was recently updated to version 2 (SSNV2) [Clette and Lefèvre, 2015], and SSNV2 was mainly used in the
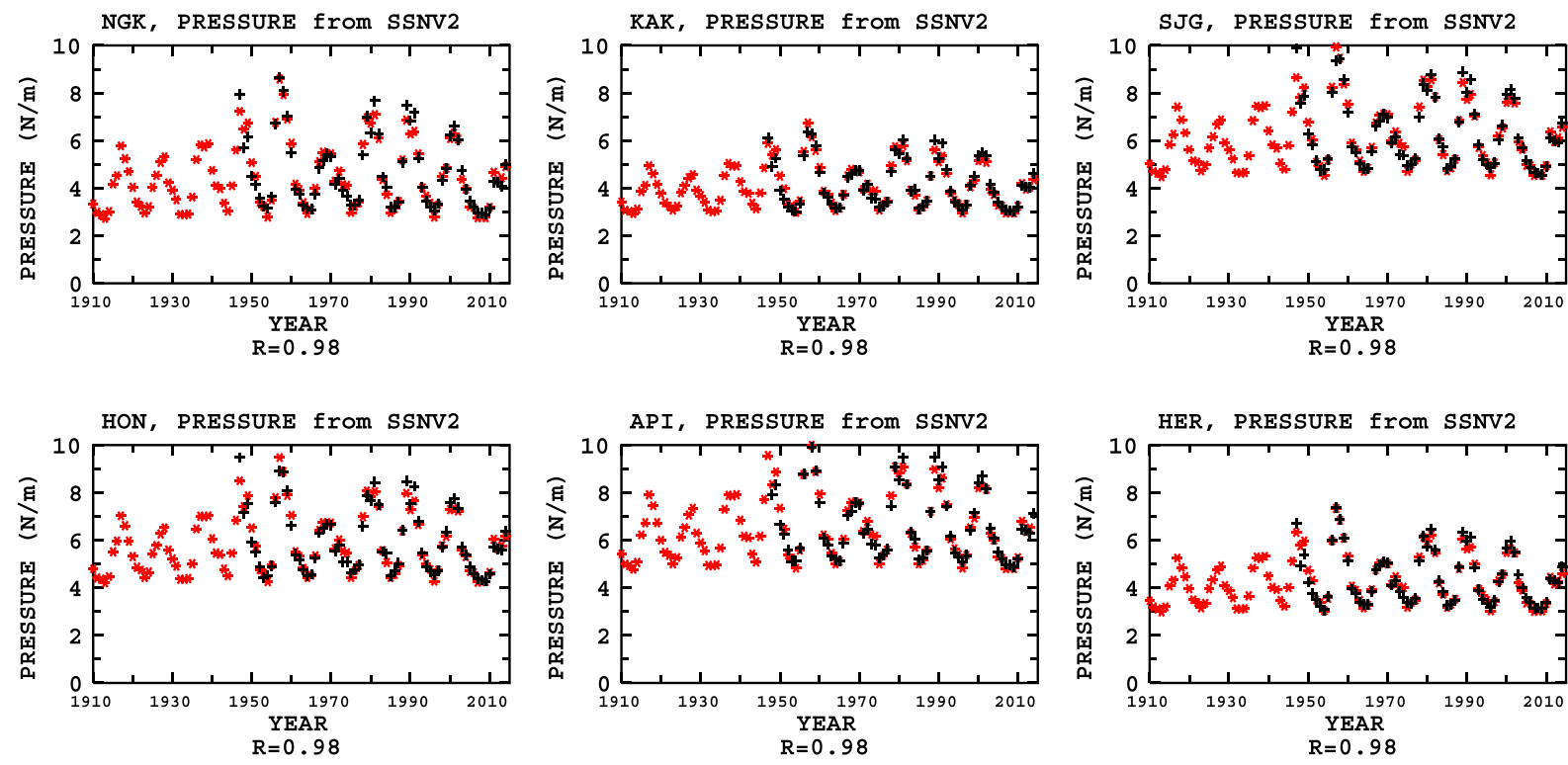

Figure 3. Same as in Figure 2 but from SSNV2. 

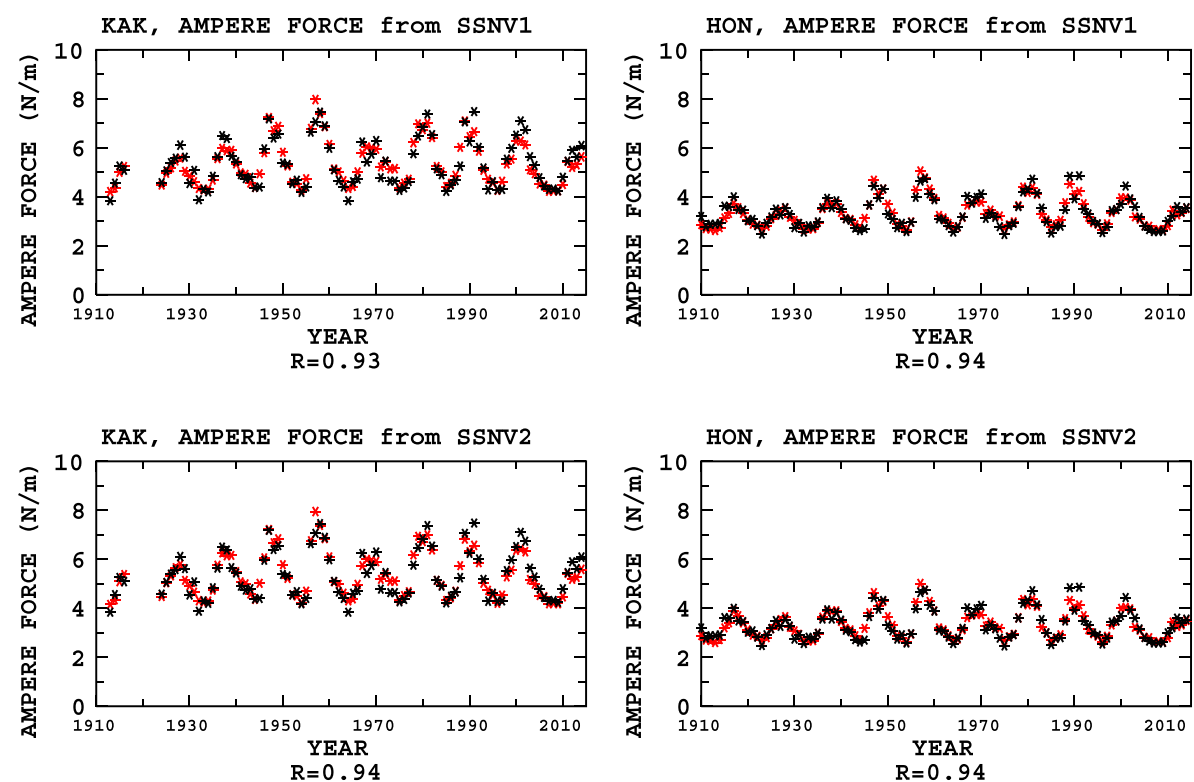

Figure 4. Model values of AF (black) and values of AF (red) accessed using linear regression coefficients from (top) SSNV1 and (bottom) SSNV2 obtained from the 1947-2010 data. $R$ indicates the correlation coefficient.

present analysis. However, the older version (SSNV1) was also used to examine the effect of the version up. Finally, a similar analysis was performed using TJ instead of AF to examine the effect of the geomagnetic main field on Sq currents.

\section{Results and Discussion}

Figure 2 shows the PD values estimated by the NRLMSISE-00 model and the assessed PD values from AF using the linear regression obtained by the data from 1947 to 2010 at each observatory. The modeled PD is fairly well reproduced by the linear regression after 1947 at all observatories, and could be highly accurate, because the correlation coefficients are between 0.85 and 0.97 . In the figure, PD before 1947 was assessed using the regression coefficients obtained from the data after 1947. This PD data are valuable for examining the long-term variation of the thermosphere, because model PD before 1947 is unavailable.

SSN has been widely used as a measure of long-term solar activity. In order to compare the predictability of SSN for PD with that of AF, Figure 3 shows the results similar to Figure 2, except that SSNV2 was used instead of AF. Since model PD is available only after 1947, the linear regression coefficients were obtained using PD and SSNV2 after 1947, and then the coefficients were used to estimate PD before 1946 to compare the results with those for AF. The obtained correlation coefficients were generally higher than those obtained for AF shown in Figure 2. One may think that SSN is better than AF for the inspection of PD, because this result shows that PD obtained using NRLMSISE-00 model can be estimated by SSN which represents the solar

Table 1. Correlation Coefficients Between AF and SSNV1 and Between AF and SSNV2 at Each Observatory for the Period of $1947-2014^{a}$

\begin{tabular}{lccccc} 
Observatory & Geomagnetic Latitude & AF-V1 & AF-V2 & TJ-V2 & $\Delta B_{z}$ \\
\hline NGK & 51.6 & 0.93 & 0.94 & 0.92 & $4.1 \%$ \\
KAK & 27.8 & 0.94 & 0.95 & 0.95 & $2.0 \%$ \\
SJG & 27.6 & 0.95 & 0.95 & 0.95 & $-26.5 \%$ \\
HON & 21.6 & 0.95 & 0.95 & 0.93 & $-6.2 \%$ \\
API & -15.1 & 0.82 & 0.83 & 0.85 & $-2.5 \%$ \\
HER & -34.0 & 0.98 & 0.98 & 0.96 & $-17.4 \%$ \\
\hline
\end{tabular}

${ }^{\mathrm{a}}$ Correlation coefficients between TJ and SSNV2 are also shown with a variation rate of the vertical component of the geomagnetic main field $\left(\Delta B_{z}\right)$. 
Acknowledgments

The author acknowledges the World Data Center for Geomagnetism, Kyoto (http://wdc.kugi.kyoto-u.ac.jp/index. html), and the observatories for obtaining the geomagnetic data (hourly values and $K p$ index) used in the present study. The data were originally supplied by Japan Meteorological Agency, U.S. Geological Survey, South African National Space Agency in South Africa, Adolf-Schmidt-Observatorium für Erdmagnetismus in Germany, and Institute of Geological and Nuclear Sciences Ltd. in New Zealand, which operate KAK, HON and SJG, HER, NGK, and API, respectively. The $a a$ index is available from http://www.geomag.bgs ac.uk/data_service/data/magnetic_indices/aaindex.html. IGRF model is available at http://www.ngdc.noaa.gov/ IAGA/vmod/igrf.html. IRI2012 model and corresponding conductivity mode are available at http://iri.gsfc.nasa.gov/ and http://wdc.kugi.kyoto-u.ac.jp/ionocond/sigcal/index.html, respectively. SSN (versions 1 and 2) and NRLMSISE-00 models are available at http://sidc.oma. be/ and http://ccmc.gsfc.nasa.gov/ modelweb/atmos/nrlmsise00.html, respectively. activity. However, it should be noted that the NRLMSISE-00 model itself refers to the solar radio flux $\left(F_{10.7}\right)$, which is a solar activity parameter, and thus, it is natural that modeled PD correlates very closely with SSN, which is in turn highly correlated with $F_{10.7}$.

On the other hand, Fukushima [1979] theoretically predicted that PD is balanced with AF. If this balance is always maintained in the thermosphere, the values of PD predicted by AF should be better than those by SSN, because PD predicted by AF can also include the effect of the long-term variation of the atmosphere caused by factors other than solar activity such as human activity. Furthermore, using AF to presume PD also has the advantage that it requires only geomagnetic field observations. In fact, SSN has recently been updated from version 1 to version 2 , and this fact shows that the old solar activity relative to recent years inferred from SSN may be erroneous, and thus, predicted PD could be used is better than that predicted by using SSN, because it is expected that PD balances with AF by Sq currents.

Next, the correlations of AF at each observatory with SSNV1 and SSNV2 were examined to check the effect of the new SSN version. Figure 4 compares the values of AF estimated from the $S q$ field in each year and those assessed from sunspot number SSNV1 (top) with SSNV2 (bottom) using linear regression obtained from the data at KAK and HON. Both SSNs can predict AF very well, but SSNV2 is slightly better at KAK. Table 1 shows the correlation coefficients for six observatories between AF and SSNV1 or SSNV2 from 1947 to 2014. A slight improvement in the correlation coefficient was found using SSNV2 at NGK and API, and it was noted that for all observatories the coefficients for SSNV1 were not larger than those for SSNV2. Although the difference is too small to be statistically significant, this suggests that SSNV2 is a better proxy for solar activity, if the effect of solar activity on AF is represented by the dependence of SSN.

If the balance between AF and PD is maintained, the intensity of $\mathrm{Sq}$ currents will be inversely proportional to the strength of the geomagnetic main field under constant PD conditions. Therefore, it is expected that the recent decrease in the geomagnetic main field by secular variation should enhance $S q$ currents, although winds blowing in the direction opposite to the pressure gradient directly affect PD, and thus, it could not be simply said that $S q$ field is inversely proportional to the main field strength. Table 1 shows also the correlation coefficients between TJ and SSNV2 together with the variation rate of the vertical component of the geomagnetic main field $\left(\Delta B_{z}\right)$. The correlation coefficients between TJ and SSNV2 were not less than those between AF and SSNV2, except at API, although the difference was small and could not be statistically significant. Since AF is affected by the main field strength and TJ is not, the inclusion of the geomagnetic main field strength was found to improve the correlation in most cases, and thus, it is suggested that the main field strength affects the $\mathrm{Sq}$ field.

\section{Conclusion}

We have examined the long-term variation of the $S q$ amplitude and effects of the solar activity.

The main results are summarized as follows:

1. The long-term trend of PD was estimated using a linear regression coefficient based on a high correlation between the AF and PD in recent years.

2. Although the difference is not statistically significant, the correlation of AF with SSNV2 is slightly higher than that with SSNV1. This suggests that the latest SSN version has improved as the index of solar activity and its effect on $\mathrm{Sq}$ and the thermosphere.

3. The correlation between AF and SSN is generally better than that between TJ and SSN. This supports the concept that the geomagnetic field also affects the intensity of $\mathrm{Sq}$ currents.

\section{References}

Celik, C. (2014), The lunar daily geomagnetic variation and its dependence on sunspot number, J. Atmos. Sol. Terr. Phys., 119, 153-161, doi:10.1016/j.jastp.2014.08.002.

Chapman, S., and J. Bartels (1940), Geomagnetism, vol. 2, Oxford Univ. Press, London.

Clette, F., and L. Lefèvre (2015), The new sunspot number: Assembling all corrections reprint, arXiv:1510.06928.

Clilverd, M. A., E. Clarke, T. Ulich, J. Linthe, and H. Rishbeth (2005), Reconstructing the long-term aa index, J. Geophys. Res., 110, A07205, doi:10.1029/2004JA010762.

Fukushima, N. (1979), Electric potential difference between conjugate points in middle latitudes caused by asymmetric dynamo in the ionosphere, J. Geomagn. Geoelectr., 31, 401-409, doi:10.5636/jgg.31.401. 
Glassmeier, K.-H., J. Vogt, A. Stadelmann, and S. Buchert (2004), Concerning long-term geomagnetic variations and space climatology, Ann. Geophys., 22, 3669-3677, doi:10.5194/angeo-22-3669-2004.

Matsushita, S. (1969), Dynamo currents, winds, and electric fields, Radio Sci., 4, 771-780, doi:10.1029/RS004i009p00771.

Miyoshi, Y., H. Fujiwara, H. Jin, H. Shinagawa, and H. Liu (2012), Numerical simulation of the equatorial wind jet in the thermosphere, J. Geophys. Res., 117, A03309, doi:10.1029/2011JA017373.

Shinbori, A., Y. Koyama, M. Nose, T. Hori, Y. Otsuka, and A. Yatagai (2014), Long-term variation in the upper atmosphere as seen in the geomagnetic solar quiet daily variation, Earth Planets Space, 66, 155, doi:10.1186/s40623-014-0155-1.

Takeda, M. (1996), Effects of the strength of the geomagnetic main field strength on the dynamo action in the ionosphere, J. Geophys. Res., 101, 7875-7880, doi:10.1029/95JA03807.

Takeda, M. (2013), Contribution of wind, conductivity and geomagnetic main field to the variation in the geomagnetic Sq field, J. Geophys. Res. Space Physics, 118, 4516-4522, doi:10.1002/jgra.50386.

Takeda, M. (2015), Ampère force exerted by geomagnetic Sq currents and thermospheric pressure difference, J. Geophys. Res. Space Physics, 120, 3847-3853, doi:10.1002/2014JA020952.

Yamazaki, Y., and M. J. Kosch (2014), Geomagnetic lunar and solar daily variations during the last 100 years, J. Geophys. Res. Space Physics, 119 , 6732-6744, doi:10.1002/2014JA020203. 